\title{
Traffic Monitoring and Incident Detection using Cellular and Early Stage VANET Technology Deployment
}

\author{
Mario De Felice $\quad$ Francesca Cuomo Andrea Baiocchi \\ Ion Turcanu Stefano Zennaro \\ Dept. of Information Engineering, Electronics and Telecommunications \\ Sapienza University of Rome, Italy, e-mail: name.surname@uniroma1.it
}

\begin{abstract}
In the current Intelligent Transportation Systems (ITS) traffic monitoring and incident detection are usually supported with mostly traditional and relatively slow reactivity technologies. In this paper we propose a new service, namely THOR (Traffic monitoring Hybrid ORiented service), able to combine two different wireless technologies and to provide real time information about vehicular traffic monitoring and incident detection. THOR relies on LTE (Long Term Evolution) and Dedicated Short Range Communication based VANETs (Vehicular ad-hoc NETworks) in a hybrid approach, which is compliant with ITS standards. This hybrid networking approach can be deployed today and can be ready for tomorrow VANET technology. We test THOR by simulations in a scenario with vehicle flows synthesized from real measured vehicular traffic traces. We provide an LTE load analysis and an assessment of incident detection capabilities. Our results are promising in terms of reactivity, precision and network traffic load sustainability.
\end{abstract}

\section{CCS Concepts}

-Networks $\rightarrow$ Network protocols; Ad hoc networks;

\section{Keywords}

Heterogeneous Vehicular Networks; Traffic Monitoring; Incident Detection

\section{INTRODUCTION}

In the recent years, a huge revolution is taking place in the automotive world. New technology has been integrated on-board of vehicles, thus making the driver aware of multiple pieces of information that makes his experience safer and richer. Unfortunately, this advancement inside the vehicle does not correspond to the same advancement outside of it. Aggregated information or macroscopic analytical instruments for Intelligent Transportation Systems (ITS) have been evolving slowly. The main example of this statement

Permission to make digital or hard copies of all or part of this work for personal or classroom use is granted without fee provided that copies are not made or distributed for profit or commercial advantage and that copies bear this notice and the full citation on the first page. Copyrights for components of this work owned by others than ACM must be honored. Abstracting with credit is permitted. To copy otherwise, or republish, to post on servers or to redistribute to lists, requires prior specific permission and/or a fee. Request permissions from permissions@ acm.org. is vehicle traffic monitoring and incident detection. An incident is intended as a car accident or any other event that obstructs the traffic flow (e.g. an object on the road). Broadly speaking, these services are supposed to be real time, but actually they have slow reactivity times with respect to vehicular traffic dynamics. Reactivity times may vary from system to system: they are in the order of tens of minutes for fixed infrastructure monitoring [18], that go down to about 5 minutes only at the cost of a very dense and expensive infrastructure. A key point is that vehicles are mostly passive in the current scenario, though we are on the brink of a major paradigm shift, boosted by the increasingly fast deployment of communications technology (e.g., DSRC, cellular, Wi-Fi, mmWave, VLC) on-board of new vehicles.

In this scenario, it is vital to provide smart paradigms to employ existing technologies and take advantage of the new ones. Although 3G/LTE represent a widespread technology, Dedicated Short Range Communication (DSRC) based Vehicular Ad-hoc NETworks (VANETs) may provide a significant solution in terms of support of innovative ITS applications. VANETs [6][7] exploit vehicle-to-vehicle (V2V) communications to ensure fast delivery of geographically scoped information, such as safety alerts, traffic efficiency messages, manufacturer-oriented applications, and comfort and entertainment data. The DSRC technology is however still in its infancy and we are still witnessing a little penetration of On Board Unit (OBU) equipment. On the other hand, it is widely recognized that cellular systems like LTE can and will play an important role to support ITS applications [1], even if the network traffic load induced by ITS applications may critically impact the cellular radio access network [17].

LTE-centric vehicular traffic monitoring schemes have been investigated, where Floating Car Data (FCD) are collected from vehicles directly, by using on-board LTE radio modules. The same LTE network is then also used to disseminate this information in an area of interest. The work in [4] gives a detailed evaluation of LTE uplink and downlink traffic load generated by specific ITS applications, including FCD collection for vehicular traffic monitoring.

In [1][10] VANET and LTE technologies are compared, identifying the strengths and weaknesses of these two approaches under different conditions (vehicular density, vehicular speed, transmission rate). On the opposite side, VANET-based traffic data collection has also been investigated (e.g., see [9][5]). An intermediate approach is represented by the employment of a heterogeneous network paradigm, identified also as a Hybrid Wireless Network [15][13][16]. The latter integrates the use of LTE cellular 
wireless communications technology with the IEEE 802.11p based VANET. The FCD collection is considered in [15]. It is optimized by designating cluster-head nodes that are responsible for reporting the FCD of their respective neighbor vehicles. A centralized architecture around the eNodeB is used in order to optimize the clusters management. The work in [13] focuses on the reduction of the impact of FCD traffic on the human-to-human traffic carried by LTE, by using a LTE-VANET heterogeneous network. Finally, [16] aims at reducing the number of concurrently active LTE channels by employing a distributed procedure that exploits the V2V DSRC multi-hop communication to elect representative nodes, which are responsible for communicating aggregated FCD via the LTE infrastructure.

The framework outlined above has lead us to propose THOR (Traffic monitoring Hybrid ORiented service). This service uses both cellular LTE and VANET with a hybrid approach to ensure the continuity of the monitoring functionality. It aims at both monitoring the traffic in real time and detect incidents in an average target time of 30/40 s, which means before a proper traffic jam is in place. THOR is LTE compliant and ETSI compatible, which means that it can run as an application on top of the GeoNetworking protocol [12] and architecture [11].

\section{THOR}

THOR collects data by sampling vehicles on the road in order to determine the traffic density, speed and infer the presence of incidents. The main logic is based on distributed timers and overhearing. Timers select the sampled vehicles, determine the LTE/VANET switching or trigger autonomous sampling or data collections. Timers are local and as a consequence THOR is fully distributed. No dedicated control packets are needed, since data packets are used to align timers.

THOR runs at the application layer. It can work in three modes: VANET mode, on top of the ETSI ITS protocol stack [11]; LTE/3G mode, when there is no VANET technology available; and Hybrid mode, where intermittent VANET connectivity is available. The default mode is VANET, but THOR automatically selects Hybrid and LTE/3G mode when needed.

\subsection{System Scenario}

We consider an urban highway, covered by the cellular network and infrastructures with RSUs. Specifically, we assume that only two RSUs are needed, $\mathrm{RSU}_{a}$ and $\mathrm{RSU}_{b}$, acting as the source and the sink of the service messages, respectively. Vehicles moving on the highway are equipped with at least a cellular terminal, possibly a DSRC OBU. On the cellular part, no synchronization or overhead messages are used, to make the service light. On the VANET part, the synchronization is provided by the RSU issuing polling messages every $T_{R S U}$, and no other overhead is needed.

All data collected in the three modes end up in a central database (ideally at the highway control room) where it is interpreted and time ordered according to the timestamps, sequence numbers and positions of the collected FCD records. In particular, the observed highway is divided into segments. The collected FCD is aggregated over time slots and geographical segments in order to estimate the current local speeds on the highway. This data is used as input to the incident detection algorithm described in Sec. 3 .

\subsection{VANET Mode}

When VANET connectivity is available, THOR collects samples from vehicles every hop interval of the multi-hop VANET radio communication. To this end, $\mathrm{RSU}_{a}$ originates a stream of messages, issuing one call for measurement collection $(\mathrm{cmc})$ message every time interval $T_{R S U}$. The $\mathrm{cmc}$ message is passed over from vehicle to vehicle by using the logic outlined below, until it reaches $\mathrm{RSU}_{b}$, that is the sink of the collected measurements. $T_{R S U}$ is chosen to satisfy the trade-off between precision and bandwidth requirements.

The header of a $c m c$ message contains the triple $\left\langle s, h, P_{X}\right\rangle$, where $s$ is the sequence number, $h$ is the hop count, and $P_{X}$ denotes the coordinates of the sending node $X$. The hop count $h$ is incremented by each subsequent relaying node. The $c m c$ messages are issued by the $R S U_{a}$ with $h$ set to 0 . The $R S U_{a}$ is in charge of incrementing the sequence number $s$ by 1 at each new message. The payload of the $\mathrm{cmc}$ messages carries the collected FCD, appended by each intermediate rely node. As soon as the $\mathrm{cmc}$ message reaches the $R S U_{b}$, its payload carries $n$ FCD records if $n$ intermediate relay nodes have forwarded the message from $R S U_{a}$ to $R S U_{b}$. Thus, THOR realizes a sampling of vehicles travelling in the highway span between $R S U_{a}$ and $R S U_{b}$.

Let $A$ be a vehicle located at a point of coordinates $P_{A}$, forwarding a message with the tuple $\left\langle s, h, P_{A}\right\rangle$ at time $t$. Any other vehicle $V$, at position $P_{V}$ within range of $A$, receives the message sent by $A$. Let $s_{V}$ be the biggest message sequence number already seen and completely dealt with by $V$. By checking that $s \leq s_{V}, V$ can discard old messages that it has already dealt with. In the following, we assume that $s>s_{V}$, i.e., the message is a new one for $V$. Let us also define the maximum hop range $R_{\max }$ as the maximum allowed distance between a source node and next-hop relay node candidates. Then, the two rules are as follows:

- Forwarding Rule. $V$ checks whether $\overline{P_{V} P_{A}} \leq R_{\max }$, where $\overline{P_{V} P_{A}}$ denotes the distance between $\bar{A}$ and $V$. If $\overline{P_{V} P_{A}}>R_{\max }, V$ ignores the message. If instead $\overline{P_{V} P_{A}} \leq R_{\max }, V$ computes a timer $\tau_{V}$ and schedules the forwarding of its copy of the message at time $t+\tau_{V}$; in case the message is eventually forwarded, its triple will be updated to $\left\langle s, h+1, P_{V}\right\rangle$.

- Inhibition Rule. If during the time interval $\left(t, t+\tau_{V}\right]$, $V$ receives another message with triple $\left\langle s, h^{\prime}, P_{V^{\prime}}\right\rangle$, i.e., with the same sequence number $s$ as the one pending to be forwarded, then $V$ performs the following check. If $h^{\prime} \geq h+1, V$ detects that another node has already forwarded the message. Hence, $V$ drops its copy of the message and gives up forwarding it. If instead $h^{\prime} \leq h$, no inhibition takes place.

The timer is the key element to enable an inhibition rule. According to the timer mechanism, the relaying node is the one that forwards the message first. For the timer we adopt a variation of the ETSI GeoNetworking protocol component named Contention Based Forwarding (CBF) [12]. The definition of the timer in our case is:

$$
\tau_{V}= \begin{cases}T_{\max }+\left(T_{\min }-T_{\max }\right) \frac{\overline{P_{V} P_{A}}}{R_{\max }}, & \overline{P_{V} P_{A}} \leq R_{\max } \\ \infty, & \overline{P_{V} P_{A}}>R_{\max }\end{cases}
$$

where $T_{\min }$ and $T_{\max }$ are constant values for minimum and maximum timer levels. 


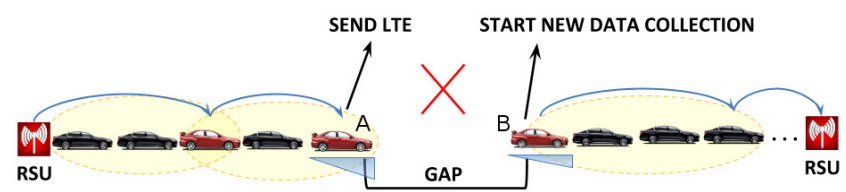

Figure 1: How THOR works: DSRC is used until it is possible; LTE is used otherwise. New $c m c$ packets are started autonomously if no connectivity with the RSU packet is available.

The payload of a $c m c$ message carries a vector $\mathbf{m}=$ $\left(m_{1}, \ldots, m_{n}\right)$ where the $i$ th element is a 3 -tuple $m_{i}=$ $\left\langle v_{i}, P_{i}, t_{i}\right\rangle$. Here $v_{i}$ is the speed of the $i$-th sampled vehicle, $P_{i}$ its geographical coordinates and $t_{i}$ a timestamp. The size of $\mathbf{m}$ depends on the number of vehicles forwarding the $\mathrm{cmc}$ message (i.e., the number of hops) from the source $R S U_{a}$ up to the sink $R S U_{b}$. The message initially issued by $\mathrm{RSU}_{a}$ has an empty payload. Each sampled vehicle, namely a vehicle acting as a forwarding node, appends its 3-tuple to the current payload of the message. When the message reaches the $\mathrm{RSU}_{b}$, it carries all $n$ collected measurements. The number $n$ is related to the average hop length and to the length of the monitored road span.

\subsection{Hybrid Mode}

Hybrid mode automatically takes over when VANET connectivity is intermittent over the highway span. Every node runs a timer $T_{L T E}>T_{\max }$. When a node $A$ forwards the $c m c$ message with sequence number $s$ and hop count $h$ on the VANET, the LTE timer $T_{L T E}$ is reset and started. If $A$ does not overhear a $\mathrm{cmc}$ message with the same sequence number $s$ and with hop count $h+1$ within $T_{L T E}$, then node $A$ deems the VANET to be disconnected and sends all the FCD records carried by the $c m c$ message to the collection server via the LTE network (see Fig. 1). In fact, if there was a node downstream $A$ with a DSRC OBU, it should be able to receive the $c m c$ message issued by $A$ and forward it within at most $T_{\max }<T_{L T E}$, since $T_{\max }$ is the maximum delay envisaged by the VANET mode of THOR.

In order to allow the VANET multi-hop node chain to restart after the gap, each node must run another timer, namely $T_{\text {InitV }}=M T_{R S U}$ (Initialize VANET). If a node $A$ does not receive any $\mathrm{cmc}$ message for a number of FCD collection periods big enough, it deems to be isolated upstream, hence it must initiate a new VANET segment. This is the condition detected by node $B$ in Fig. 1 . As the timer $T_{\text {InitV }}$ expires, node $B$ will schedule a new $c m c$ message, carrying the triple $\left\langle s_{B}+1,1, P_{B}\right\rangle$, where $s_{B}$ is the last $c m c$ sequence number received by $B$ through the VANET. The scheduled message undergoes the timer based forwarding procedure described in the VANET mode, to avoid duplicated messages. It is important to notice that $B$ will be the first node among its neighbours whose $T_{\text {InitV }}$ elapses, since $T_{\text {InitV }}$ is reset on reception.

\subsection{LTE Mode}

The third operation mode is LTE only. The LTE mode is triggered automatically when the Hybrid mode can no longer be applied, because of total lack of the VANET. If a vehicle node $B$ does not receive $c m c$ messages for at least $T_{\text {InitV }}$, it starts its own VANET collection segment. However, if $B$ does not overhear any forwarding operation within $T_{L T E}$, it concludes to be an isolated node over the DSRC channel, hence it sends its own FCD via LTE and resets $T_{\text {InitV }}$. This simple timer provision makes any vehicle node seamlessly move to LTE whenever the VANET is completely absent. Coupled with the other two modes, this implies a graceful adaptation of the FCD collection procedure to the market penetration rate of the DSRC OBU equipment.

\section{THE INCIDENT DETECTION}

The monitoring mechanism described in Sec. 2 allows the collection of FCD from vehicles. This information is then used to detect the presence of incidents on the monitored highway. We have developed a 2-phase algorithm called Local ObstrUction Detector (LOUD), based on sampled FCD from the observed highway. LOUD can work with any FCD collection protocol: we have developed LOUD based on THOR, independently on THOR's operation mode.

The main idea behind LOUD is that we look for the number of vehicle velocity samples that fall below a threshold in a given time interval (slowdown event). The threshold level and the interval duration must be suitably calibrated. When the slowdown event is detected, that does not necessarily mean that an accident has occurred. Such a behaviour can be caused by a shock wave or an occasional phenomenon. Because of that, the slowdown event only triggers an Alarm Phase (AP). The decrease trend of vehicles' velocities must be confirmed over a period of time. If that is the case, the incident detection is confirmed and an alarm to the vehicles travelling ahead of the impacted section is sent, along with an alternate route to avoid the incident area, if possible (Incident Phase, IP).

The operation of LOUD is so organised. Time is divided into discrete intervals of duration $T_{R S U}$, i.e., the period of FCD collection request issuing. An instance of the algorithm is run for each monitored highway segment. Let $z_{s}[i]$ be the number of vehicles whose speed is less than a threshold $v$ in the $s$-th segment during the $i$-th time interval of duration $T_{R S U}$. The LOUD algorithm consists of a state machine with the following states or phases: the Normal Phase $(N P)$, the Pre-alarm Phase $k\left(P P_{k}, k=1, \ldots, n\right)$, the Alarm Phase $(A P)$, and the Incident Phase $(I P)$.

During the sojourn in the NP, the following test is checked in each time interval:

$$
e+\sum_{i=k-2 N+1}^{k-N} z_{s}[i]<\eta \sum_{j=k-N+1}^{k} z_{s}[j]
$$

where $N$ is the number of consecutive time periods of duration $T_{R S U}$ over which the sequence $z_{s}[i]$ is smoothed out (averaging window size), $\eta$ is the system sensitivity factor, and $e$ is a tolerance coefficient to protect the system from false positive when few vehicles slow down.

As soon as the condition of eq. (2) is verified, this means that the number of slow or still vehicles has grown anomalously in the $s$-th highway segment over the last $N$ time intervals. Then, the LOUD state machine moves to the $P P_{1}$ state, i.e., to the first pre-alarm phase, where the test of eq. (2) is repeated again. In general, in the $P P_{k}$ state, the test in eq. (2) is checked: if satisfied, the state moves to $P P_{k+1}$, while it goes back to $N P$ in case the test fails. If the validity of the condition in eq. (2) is confirmed for $n$ consecutive times, then LOUD enters definitely in the $A P$ 
state. The delay for declaring the alarm condition from the onset of the critical conditions is $n T_{R S U}$.

When in the $A P$ phase, LOUD checks a different condition, stated as:

$$
\sum_{i=k-2 N+1}^{k-N} z_{s}[i]-e \leq \sum_{j=k-N+1}^{k} z_{s}[j]
$$

The eq. (3) verifies that the number of slow vehicles is non-decreasing over time with respect to the one that triggered the onset of the alarm, within the tolerance constant $e$. Hence, this test helps discarding short term slow downs. Once the AP is reached for the given sector, LOUD detects an incident and moves to the $I P$ only if the condition in eq. 3 is confirmed for $k=\left\lceil T_{p e} / T_{R S U}\right\rceil$ consecutive $T_{R S U}$ intervals. Once in the $I P$, when the condition in eq. (3) becomes false, the incident alarm is switched off.

In [2] LOUD parameters have been tuned in order to optimize the trade-off between system precision (low false positive rates), efficiency (hit ratio of successfully detected incidents) and incident detection delay.

\section{SIMULATION SCENARIO}

In order to provide an accurate estimation and a realistic challenge for THOR and LOUD, we have put in place a study of about 104 millions of real GPS traces collected by about 80.000 vehicles travelling within the metropolitan area of Rome, Italy. We focused on a subset of 50.220 vehicles travelling on a $68 \mathrm{~km}$ ring-shaped motorway that surrounds the city of Rome, named Grande Raccordo Anulare (GRA). It collects and distributes long-haul traffic entering and exiting the city.

In order to analyse the data, we have divided the GRA in 29 different sectors of length $L_{j}, j=1, \ldots, 29$, where the main exit ramps are the starting and ending points of each sector. The data samples of the most intense four hour traffic ( $3 \mathrm{pm}$ and $7 \mathrm{pm}$ ) have been inferred to the universe of vehicles by assuming a random uniform sampling of the GPS-equipped vehicles that were source of floating car data. Let $\Delta t$ be the sampling interval (30 s in our study), $v_{i}$ the detected speed values of vehicle $i$ during the time interval $\left[t_{1}, t_{2}\right], n_{j}$ the estimated number of vehicles travelling on the $j$-th sector, $g_{j}\left(t_{1}, t_{2}\right)$ the number of detected GPS signals on the $j$-th sector during the observation time interval $\left[t_{1}, t_{2}\right]$, $L_{j}$ the length of the $j$-th sector, $a$ the probe vehicles penetration rate ( $a \approx 2.3 \%$ in our study) and $q_{j}$ the estimated flow on the $j$-th sector. Then:

$$
n_{j}=\frac{1}{L_{j}} \sum_{i=1}^{g_{j}\left(t_{1}, t_{2}\right)} v_{i} \Delta t, \quad q_{j}=\frac{n_{j}}{a\left(t_{2}-t_{1}\right)}
$$

The above inference relations have been applied to estimate the average flow $q_{j}$ on each sector $j$ in the peak period. Given the link flows on each road sector, the OriginDestination (O-D) traffic matrix between the 29 junctions has been estimated as detailed in [2]. The above vehicles flows have been used as an input to generate a vehicular simulation using SUMO [3], that provides the possibility to import a real road map and to inject the desired vehicles flows in it. SUMO allows to mimic the real vehicle interactions through car following and lane changing models.

On top of the vehicular simulation provided by SUMO, we have used NS-2 [8] as a network simulator, to reproduce

\begin{tabular}{l|l}
\hline Parameter & Value \\
\hline Road length $(\mathrm{km})$ & 68.2 \\
Number of lanes per travelling direction & 3 \\
Average vehicle density (veh/km) & 31.02 \\
SUMO simulation duration per scenario (s) & 3600 \\
Network simulation duration per scenario (s) & 400 \\
Frequency of generated messages (msg/s) & 1 \\
DSRC $R_{\max }(\mathrm{m})$ & 827 \\
MAC, PHY parameters & IEEE 802.11p \\
$N, e, \eta, T_{p e}(\mathrm{~s})$ & $5,1,1.7,15$ \\
$T_{\max }, T_{L T E}, T_{\text {initV }}(\mathrm{s})$ & $0.1,0.2,3.5$ \\
\hline
\end{tabular}

Table 1: Simulation parameter values

the features of the radio channel, the IEEE 802.11p PHY and MAC protocols and the FCD collection logic defined in Sec. 2, that we have implemented into NS2.

We assume that all vehicles in our experiment can send their FCD through the cellular network (LTE or 3G). As for the DSRC penetration rate $p$, it ranges between $0 \%$ and $100 \%$. We have run several simulations with different market penetration rate conditions, in order to cope with the different conditions in terms of VANET coverage that we can have during the first stages of deployment. Since the inhibition rule cannot be applied to vehicles having LTEonly technology on-board, the later send their FCD via the cellular network with a probability equal to the fraction of sampled vehicles when assuming $p=100 \%$. Each simulation has a stabilization phase in which data is not collected, while the map is populated up to a steady state. Then an observation time window of about $400 s$ starts, where THOR is run and statistics on its performance are estimated.

In our study, since the scenario is a ring-shaped motorway, we have chosen to collapse $R S U_{a}$ and $R S U_{b}$ into the same physical RSU, thus leading to the coverage of $68 \mathrm{~km}$ with just one RSU.

\section{PERFORMANCE RESULTS}

The aim of the performance evaluation is to assess: i) the impact of the DSRC market penetration rate on the accuracy of the traffic monitoring (Sec. 5.1); ii) the off-loading potential of the cellular network entailed by the mixed operations of THOR (Sec. 5.2); iii) the ability of the incident detection algorithm to react in real time (Sec. 5.3).

The values of the numerical parameters used in the simulations are listed in Tab. 1.

\subsection{Traffic Monitoring}

The traffic monitoring is exemplified by estimating the mean velocity in each highway sector in real time. To this end, one $c m c$ message is issued by the RSU every $T_{R S U}=$ $1 s$ and the collected velocity samples for each one of the 2 driving directions of the GRA are integrated over a time window of $K T_{R S U}$, with $K=5$ to obtain the estimated velocity value $\hat{V}_{k}(n)$ of sector $k, k=1, \ldots, 29$ in the $n$-th monitoring interval $\left[n K T_{R S U},(n+1) K T_{R S U}\right)$. The average velocity percent error is defined as:

$$
\epsilon_{k}=100 \cdot \frac{1}{S} \sum_{n=1}^{S} \frac{\left|\hat{V}_{k}(n)-V_{k}(n)\right|}{V_{k}(n)}
$$

where $V_{k}(n)$ is the true value of the mean velocity of sector $k$, obtained by averaging the velocities of all vehicles in the $k$-th sector during the polling interval $\left[n K T_{R S U},(n+1) K T_{R S U}\right)$, and $S$ is the number of monitoring intervals. Notice that 


\begin{tabular}{l|l|l|l|l|l}
\hline Pen. Rate $\boldsymbol{p}(\boldsymbol{\%})$ & $\mathbf{1 0 0}$ & $\mathbf{7 5}$ & $\mathbf{5 0}$ & $\mathbf{2 5}$ & $\mathbf{0}$ \\
\hline Error $\epsilon_{k}(\%)$ & 6.5 & 6.3 & 3.6 & 3.5 & 3.4 \\
\hline
\end{tabular}

Table 2: Speed estimation error for different percentage of DSRC-equipped vehicles

$V_{k}(n)$ represents the ground truth obtained from SUMO, that is, the true mean vehicle velocities provided by the collected real GPS traces.

The average velocity percentage error for different penetration rates of DSRC-equipped vehicles is displayed in Table 2 . In case $p=100 \%$, only the VANET mode is used, since the vehicle density is high enough that no disconnection of the VANET arises. In this case the error is about $6.5 \%$. This performance level strikes an optimal tradeoff in terms of network load vs effectiveness of the incident detection algorithm LOUD, that exploits the collected velocity data. The results that go from $75 \%$ to $25 \%$ market penetration rates in Table 2 depict the behaviour of the Hybrid Mode. In such a modality, when the VANET coverage is missing, LTE takes over. It can be noticed that the mean error percentage drops down as we approach to LTE/3G only monitoring. Such behaviour can be explained by the fact that the CBF [12] protocol tends to sample at fixed geographical positions in time. This structured sampling might not always reflect the actual mean velocity distribution. The best result is in the LTE/3G only mode, when the VANET connectivity is completely missing, with an average error of $3.4 \%$. In this case every vehicle sends periodically its own FCD with a probability equal to the fraction of sampled vehicles when assuming $p=100 \%$. This leads to a better geographical distribution of sampled vehicles, which gives a lower error.

\subsection{LTE/3G Load Analysis}

Our baseline reference is the exhaustive collection of velocity samples from vehicles with a time period of $3.5 \mathrm{~s}$. This poses the maximum load on the cellular network in our scenario. As the market penetration rate of OBU equipment increases, a growing off-load of the cellular network is possible, since fewer vehicles report on their velocity, those sampled by the VANET component of THOR.

From the results obtained by simulation the offered load to the mobile network was evaluated. The key parameter employed to estimate the average number of bits to Base Stations is the vehicle sampled FCD. In our simulations the length of a single FCD packet is 44B (12B for the header and $32 \mathrm{~B}$ for the payload).

Fig. 2 depicts the normalized average offered load due to the collection of the periodic messages of the LTE compo-

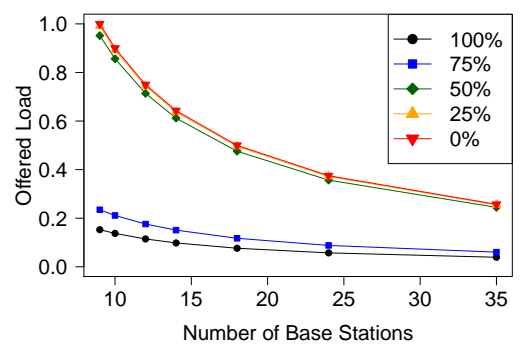

Figure 2: Normalized LTE average offered load by THOR as a function of the number of BSs.

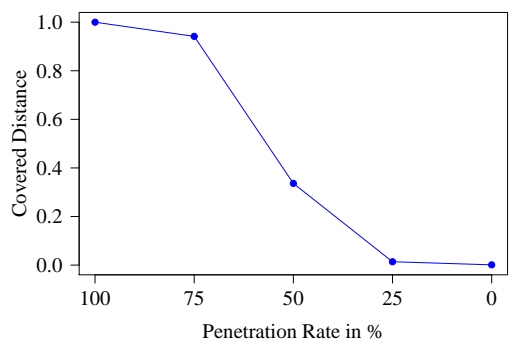

Figure 3: Normalized covered distance using CBF [12] protocol as a function of the percentage of DSRC-equipped vehicles.

nent of THOR, as a function of the number of base stations covering the GRA highway area. We have assumed that the base stations are uniformly distributed along the highway. Every point on the graph is normalized with respect to the maximum offered load, which in our case is when considering 9 base stations and $p=0 \%$. It can be noticed that the lowest average offered load to the cellular network is given by a penetration rate of $100 \%$. When $p=75 \%$ the offered load slightly increases, but it's still close to the $p=100 \%$ case, which can be explained by the fact that with a penetration rate of $75 \%$ the density of OBU equipped vehicles is high enough to have very few VANET disconnections and isolated vehicles.

From Fig. 2 we can notice that a big gap separates the load curves for low penetration rates and those for high penetration rates (greater or equal than $75 \%$ ). This is typical of the non-linear transition of the VANET connectivity probability taking place as the mean density of the DSRC equipped vehicles decreases. The mean covered distance of the FCD collection protocol by sampling can be estimated by an analytical model under the assumption of Poisson vehicle spatial distribution (see [14]). The results are shown in Fig. 3. In can be seen that an abrupt shortening of the mean traveled distance of messages is incurred when the market penetration ratio drops from $75 \%$ down to $50 \%$, consistent with the curves of Fig. 2.

Table 2 and Fig. 2 show that even if THOR slightly increases the average speed estimation error for high DSRC penetration rates, which is however acceptable for a wide range of applications, it gives a notable decrease in terms of offered load to the cellular network.

\subsection{Real Time Incident Detection}

Our proposed incident detection algorithm, LOUD, uses data collected by THOR. In Fig. 4 we can see the results in terms of percentage of detected incidents ( $y$ axis) over all the simulated incidents as a function of the time it takes LOUD to converge and reveal the incident. This time is comprehensive of LOUD's two phases.

Although very fast, the convergence could be even faster with different tuning of the parameters described in Sec. 3 and reported in Tab. 1. Having a slightly slower convergence time (minimum 20s) is the price to pay to keep precision over $99 \%$, which is a vital functional requirement for an automated real time application. That is why those parameters have been optimized to guarantee a precision not less than $99 \%$ in [2].

In Fig. 4, it is possible to notice that only $30 s$ are needed to detect $50 \%$ of the incidents, regardless of the penetration 


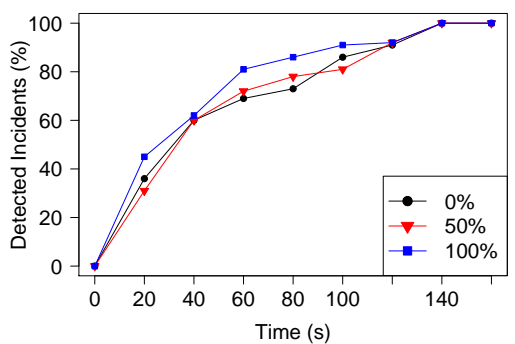

Figure 4: Percentage of detected incidents sampled every 20s.

rate. This is an outstanding result and clearly shows that half of the incidents can be detected in about $30 \mathrm{~s}$ through LOUD, which is at least an order of magnitude faster than any other approach, as detailed in Sec. 1.

We simulated different kind of incidents on different sectors of our map, in order to achieve heterogeneity and allow us to analyse and tune our algorithm to recognize different types of incidents. The incidents consist in simulated collision and subsequent obstruction of a number of lanes: since the total number of lanes in our scenario is 3 , for every incident we have 3 simulations, with 1 obstructed lane (the slowest lane), then 2 (still the slowest ones) and finally all of them. Every segment of GRA has at least one incident and thus 3 simulations, for a total of 87 different types of incident, with different vehicle loads on the segment.

The general trend is pretty much independent with respect to the DSRC penetration rate and this means that the algorithm is robust with respect to the VANET deployment and would perfectly work in LTE only mode today. This also proves that the error rates discussed in Sec. 5.1 are the right trade-off for the automatic algorithm to work properly. The slopes evolve with about the same behaviour and this happens because LOUD is independent with respect to the technology used to collect data.

\section{CONCLUSIONS}

In this paper we propose a traffic monitoring algorithm, THOR, that combines the LTE/3G and the VANET technology in an hybrid and standard compliant approach. We used this application to gather data to feed LOUD, an application capable of detecting incidents in real time. We have tested both algorithms in simulations based on real world GPS traces over a $68 \mathrm{~km}$ long urban highway. We showed how THOR collects the needed information with a very low error and a relatively low impact on the existing LTE/3G network and how it is capable of exploiting even small percentages of VANET presence, thus making it ready for tomorrow's technology advancement. We showed how LOUD translates the collected information in automated real time incident detection, being able to detect and react on most of the incidents in about $40 \mathrm{~s}$.

\section{REFERENCES}

[1] G. Araniti, C. Campolo, M. Condoluci, A. Iera, and A. Molinaro. LTE for Vehicular Networking: A Survey. IEEE Communications Magazine, 51(5):148-157, May 2013.

[2] A. Baiocchi, F. Cuomo, M. D. Felice, and G. Fusco. Vehicular Ad-Hoc Networks sampling protocols for traffic monitoring and incident detection in Intelligent Transportation Systems. Transportation Research Part C: Emerging Technologies, 56:177-194, 2015.

[3] M. Behrisch, L. Bieker, J. Erdmann, and D. Krajzewicz. SUMO-Simulation of Urban MObility-an Overview. In SIMUL 2011, pages 55-60, 2011.

[4] J. Calabuig, J. Monserrat, D. Gozalvez, and O. Klemp. Safety on the Roads: LTE Alternatives for Sending ITS Messages. Vehicular Technology Magazine, IEEE, 9(4):61-70, December 2014.

[5] Y. Dieudonne, B. Ducourthial, and S. Senouci. COL: A data collection protocol for VANET. In $I V$, 2012 IEEE, pages 711-716, June 2012.

[6] P. d'Orey and M. Ferreira. ITS for Sustainable Mobility: A Survey on Applications and Impact Assessment Tools. ITS, IEEE Transactions on, 15(2):477-493, April 2014.

[7] F. Dressler, H. Hartenstein, O. Altintas, and O. Tonguz. Inter-vehicle communication: Quo vadis. Communications Magazine, IEEE, 52(6):170-177, June 2014.

[8] K. Fall and K. Varadhan. ns Notes and Documentation, 2000.

[9] M. Gramaglia, M. Calderon, and C. Bernardos. ABEONA Monitored Traffic: VANET-Assisted Cooperative Traffic Congestion Forecasting. IEEE Vehicular Technology Mag., 9(2):50-57, June 2014.

[10] Z. Hameed Mir and F. Filali. LTE and IEEE 802.11p for vehicular networking: a performance evaluation. EURASIP JWCN, 2014(1), 2014.

[11] E. T. S. Institute. ETSI EN 302636 -3 v1.2.1; Intelligent Transport Systems (ITS); Vehicular Communications; GeoNetworking; Part 3, Dec. 2014.

[12] E. T. S. Institute. ETSI TS 302 636-4-1 v1.2.1; Intelligent Transport Systems (ITS); Vehicular Communications; GeoNetworking; Part 4, July 2014.

[13] S. Jia, S. Hao, X. Gu, and L. Zhang. Analyzing and relieving the impact of FCD traffic in LTE-VANET heterogeneous network. In ICT 2014, pages 88-92, May 2014.

[14] D. Miorandi and E. Altman. Connectivity in one-dimensional ad hoc networks: A queueing theoretical approach. Wireless Networks, 12(5):573-587, 2006.

[15] G. Remy, S.-M. Senouci, F. Jan, and Y. Gourhant. LTE4V2X: LTE for a Centralized VANET Organization. In GLOBECOM, 2011 IEEE, pages 1-6, December 2011.

[16] P. Salvo, I. Turcanu, F. Cuomo, A. Baiocchi, and I. Rubin. LTE floating car data application off-loading via VANET driven clustering formation. In WONS 2016, pages 1-8, January 2016.

[17] S. Uppoor and M. Fiore. Characterizing Pervasive Vehicular Access to the Cellular RAN Infrastructure: An Urban Case Study. IEEE Trans. on Vehicular Technology, 64(6):2603-2614, June 2015.

[18] Z.-z. Yang, L. Gao, Z.-y. Gao, Y.-f. Sun, and S.-m. Guo. Traffic Incident Analysis on Urban Arterials Using Extended Spectral Envelope Method. arXiv preprint arXiv:1507.00224, 2015. 